Die Herausgeber und wohl auch fast alle Autoren bedanken sich sehr herzlich bei den Gutachtern, die einen Teil ihrer Arbeitskraft für das Zustandekommen der Beiträge in dieser Zeitschrift, so wie sie sind bzw. nicht sind, investiert haben. Während der Entstehungszeit von Band 6 haben referiert:
D. Bartmann, München
J. Gruber, Hagen
M. Bitz, Hagen
H. Hamacher, University
H. Müller-Merbach, Kaiserslautern
K.-P. Schuster, Hamburg
H. H. Bock, Aachen of Florida
P. Naeve, Bielefeld
K. Brockhoff, Kiel
F. Hanssmann, München
W. Buck, Karlsruhe
H. Hauptmann, Hamburg
H. H. Oberle, München
O. Opitz, Augsburg
$\mathrm{K}$. Hinderer, Karlsruhe
D. Ohse, Frankfurt
W. Bühler, Dortmund
D. Hochstädter, Frankfurt
H. Paessens, Karlsruhe
A. Hordijk, Leiden
U. Derigs, Bonn
G. Hübner, Hamburg
H. Rinne, Gießen
J. Rosenmuiller, Bielefeld
D. Dietrich, Ottobrunn
H. Isermann, Bielefeld
H. Rothacker, Dortmund
H. Seelbach, Hamburg
W. Domschke, Hamburg
H. J. Drumm, Regensburg
M. Jünger, Augsburg
B.-M. Sahling, Karlsruhe
M. Schäl, Bonn
U. Eckhardt, Hamburg
D. Kalin, Ulm
P. Kall, Zürich
S. Schaible, Edmonton
K. Floret, Oldenburg
J. Kindler, Darmstadt
H. Schellhaas, Darmstadt
R. Selten, Bielefeld
W. Sendler, Trier
O. Spaniol, Frankfurt
G. Franke, Konstanz
H.-P. Kistner, Bielefeld
G. Schmidt, Saarbrücken
W. Krabs, Darmstadt
R. Schmidt, Kiel
P. Stahlknecht, Osnabrück
H. Steckhan, Regensburg
K.-W. Gaede, München
G. Latouche, Brüssel
Chr. Schneeweiß, Mannheim
D. Steinhausen, Münster
L. Streitferdt, Hamburg
K. Geihs, Frankfurt
M. Meyer, Nürnberg
H. Schneeweiß, München
S. H. Tijs, Nijmegen
M. Vlach, Prag
J. van der Wal, Eindhoven
K.-H. Waldmann, Berlin
A. Wanka, Köln
R. Weber, Gelsenkirchen
J. Wessels, Eindhoven
J. Wilhelm, Bonn
P. Gorny, Oldenburg
E. Zemel, Northw. University

\title{
Ausschreibung eines Studentenpreises
}

Der Vorstand der Deutschen Gesellschaft für Operations Research (DGOR) lädt zu einem studentischen Wettbewerb ein, bei dem Diplomarbeiten auf dem Gebiet des Operations Research ausgezeichnet werden sollen. Sie werden von einer vom Vorstand eingesetzten Jury ausgewählt.

Die Autoren der prämiierten Arbeiten werden eingeladen, auf der DGOR-Jahrestagung 1985 in Hamburg über ihre Ergebnisse zu berichten, und zwar an zentraler Stelle des Programms. In einer entsprechend gekürzten Fassung werden die Ergebnisse ferner in den Proceedings der Tagung veröffentlicht. Die DGOR übernimmt die Reisekosten der Autoren (Bahn, 2. Klasse, von Berlin aus Flug) sowie die üblichen Aufenthaltsspesen.

Teilnahmeberechtigt sind Studentinnen und Studenten deutschsprachiger Hochschulen aller Fachrichtungen unter den folgenden Bedingungen:

- Die Diplomarbeiten müssen zwischen dem 1. Januar und dem 31. Dezember 1984 abgeschlossen sein.

- Der Autor der Arbeit soll in der Lage und bereit sein, seine Ergebnisse mündlich während der DGOR-Jahrestagung vom 11.-13. September 1985 in Hamburg vorzutragen.

- Die Arbeiten sollen über den betreuenden Hochschullehrer eingereicht werden.

- Die Arbeiten sind mit einer Zusammenfassung von maximal 4 Seiten einzureichen. Die Zusammenfassung soll die Anschriften und Telefonnummern des Verfassers und des betreuenden Hochschullehrers enthalten.

- Die Arbeiten sind bis spätestens 28. Februar 1985 zu senden an

Prof. Dr. R. Schmidt

Christian-Albrecht-Universität

Institut für Betriebswirtschaftslehre

Olshausenstraße 40

D-2300 Kiel

Telefon: (0431) 8803119

DEUTSCHE GESELLSCHAFT FƯR OPERATIONS RESEARCH (DGOR)

gez. K.P. Schuster (Vorsitzender) 